\author{
Military Technical College \\ Kobry El-Kobbah, \\ Cairo, Egypt.
}

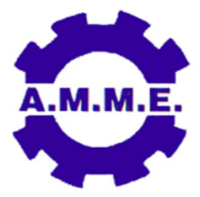
$16^{\text {th }}$ International Conference on Applied Mechanics and Mechanical Engineering.

\title{
NUMERICAL MODELING OF HYDROGEN PREMIXED COMBUSTION
}

\author{
M. A. Abdel-Raheem ${ }^{\star}$, S. S. Ibrahim ${ }^{\star *}$ and W. Malalasekera ${ }^{\star \star \star}$
}

\begin{abstract}
The scarcity of oil and gas resources through the whole world put the scientific community in a challenge to secure an alternative source of fuel. The tendency to go for hydrogen as a clean fuel and an energy carrier brings in safety issues that have to be addressed before any wide consent can be achieved. In this regard, availability of accurate modelling techniques is very useful. This paper presents Large Eddy Simulations (LES) as a modelling technique for propagating turbulent premixed flames of hydrogen-air mixtures in a laboratory scale combustion chamber. A Dynamic Flame Surface Density (DFSD) model where the reaction rate is combined with the fractal analysis of the flame front structure, is employed and tested. The fractal dimension is evaluated dynamically based on the instantaneous flow field. The main focus of the current work is to establish the LES technique as a good numerical tool to calculate turbulent premixed hydrogen flames having an equivalence ratio of 0.7 . Developing this capability has practical importance in understanding different combustion phenomena like explosion hazards, internal combustion engines and gas turbine combustors. The results obtained with the DFSD model compare well with published experimental data. A detailed analysis is planned for further validation for the LES-DFSD model for different flow geometries with hydrogen combustion.
\end{abstract}

\section{KEY WORDS}

Hydrogen, LES, Dynamic Flame Surface Density, Premixed Flames, Reaction Rate

\footnotetext{
PhD Student, Wolfson School of Mechanical and Manufacturing Engineering, Loughborough University, Loughborough, UK.

** Senior Lecturer, Department of Aeronautical and Automotive Engineering, Loughborough University, Loughborough, UK.

*** Professor, Wolfson School of Mechanical and Manufacturing Engineering, Loughborough University, Loughborough, UK.
} 


\section{INTRODUCTION}

The lack of fossil fuel in addition to the harmful environmental impact has generated an interest in hydrogen as a suitable replacement fuel and an energy carrier. This is because of its availability from many resources and zero carbon emissions. However, to enable its widespread usage in practical applications, tough challenges must be overcome regarding hydrogen and further studies are needed to develop an improved understanding of the issues affecting the generation, storage, distribution as well as combustion and accidental explosions. A key parameter behind these issues is the thermo-physical properties of hydrogen as it is very reactive and explosive gas in nature. The objective of the present work is to develop a numerical technique for computing the overpressures resulting from hydrogen combustion.

This current work uses the large eddy simulation technique to calculate the structure of lean hydrogen flames propagating inside a vented chamber while interacting with solid obstructions. Large Eddy Simulation (LES) technique is now accepted as a reliable computational tool to study turbulent flames both premixed and nonpremixed [1-6] although its additional computational cost (i.e. computational time and resources). A key advantage of LES lies in its ability to compute the complex dynamics of turbulent flows and resolve transient processes such as flame propagation, instability, extinction, as well as ignition. The computational cost and accuracy of LES solutions lie between Direct Numerical Simulation (DNS) and Unsteady Reynolds Averaged Navier-Stokes (URANS) techniques. A vital challenge to the advancement of LES lies in the development of suitable Sub-Grid-Scale (SGS) models, which are capable of representing combustion over a wide range of flow conditions.

As the reaction zone thickness of the premixed flame to be resolved is thin, with a characteristic length scale much smaller than a typical LES filter width, an appropriate SGS model is vital to account for chemical reaction. Earlier studies $[7,8]$ using DFSD model based on laminar flamelets were promising in predicting key characteristics of propagating turbulent premixed flames with built-in solid obstructions. The work presented in this paper is a continuation of previous research $[7,8]$ where progress has been made in the development of a Dynamic Flame Surface Density (DFSD) model to account for the SGS chemical reaction rate. Here, the same strategy is applied where the DFSD model is used to simulate transient turbulent premixed flames of a hydrogen-air mixture with equivalence ratio of 0.7 , propagating in a small vented chamber having 3 baffles and a square solid obstacle. The small scale combustion chamber [9] offers the capability to configure various geometries with a range of turbulent flow conditions. The LES simulations use a grid independent resolution and the results are validated against the experimental data of Masri et al. [9].

\section{TEST CASE}

The experimental chamber used in this study was developed by the University of Sydney, Australia [9]. The combustion chamber has dimensions of $50 \times 50 \times 250$ $\mathrm{mm}$ and consists of 3 baffle plates located at equidistance and a solid square obstacle of size $12 \times 12 \mathrm{~mm}$ at about $96 \mathrm{~mm}$ from the ignition end (Figure 1a). In the 
experiments the hydrogen-air mixture enters the atmospheric pressure chamber, where the mixture is allowed to settle before being ignited by focusing the infrared output from a Nd:YAG laser $2 \mathrm{~mm}$ above the base. A hinged flap on top of the chamber contains the mixture during fill time prior to ignition. This flap is raised just before ignition and is maintained through the combustion process to allow venting.

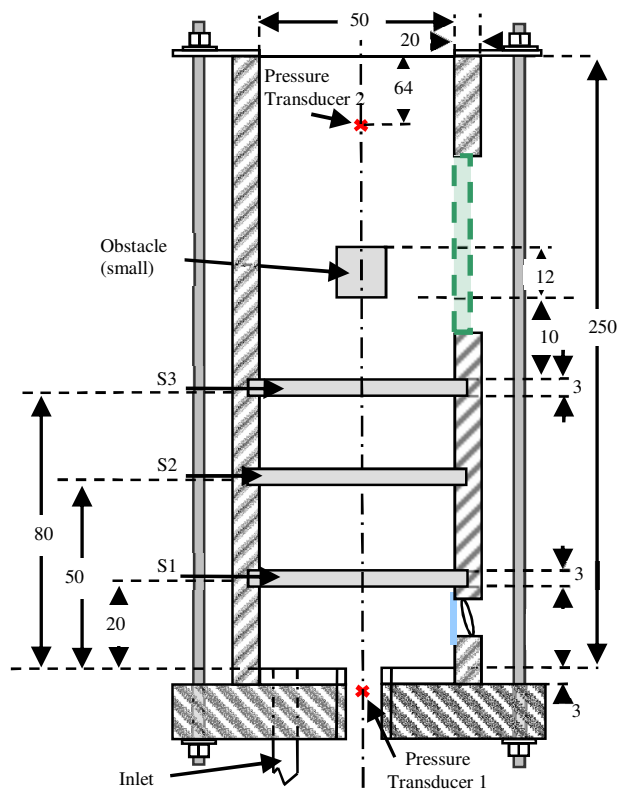

(a)

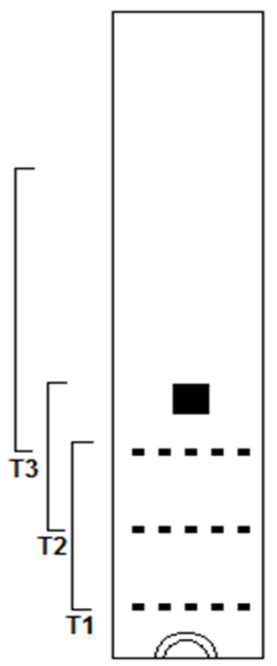

(b)

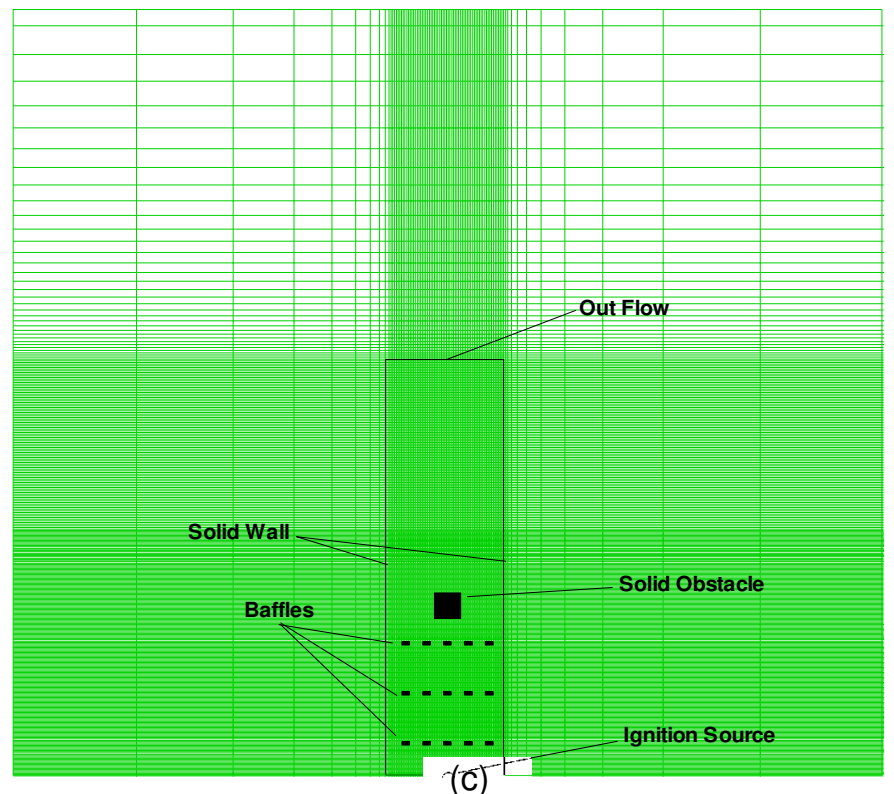

Fig. 1. (a) Schematic diagram for the Sydney combustion chamber (dimensions in mm). (b) The three imaging tiers used to capture the maximum viewable height [9]. (c) Illustration of the computational domain with the combustion chamber and the obstacles are superimposed over grid resolution. 


\section{THE COMBUSTION MODEL}

The modelling of the mean chemical reaction rate in turbulent premixed flames is very challenging especially in LES, due to its non-linear relation with chemical and thermodynamic states. It is often characterized by propagating thin reaction layers thinner than the smallest turbulent scales. In the present simulations, the SGS chemical reaction rate is accounted for by using a recently developed DFSD model $[2,7,8]$ modified for hydrogen flames. Brief details of the model are given here. More details can be found elsewhere [7].

The mean SGS chemical reaction rate $\left(\overline{\dot{\omega}}_{\mathrm{c}}\right)$ is the source term, which is modelled by following the laminar flamelet approach as:

$$
\overline{\dot{\omega}}_{c}=\rho_{u} u_{L} \bar{\Sigma}
$$

Where $\left(\rho_{u}\right)$ is the density of unburned mixture, $\left(u_{L}\right)$ is the laminar burning velocity calculated from [11], and $(\Sigma)$ is the flame surface density (FSD). The filtered flame surface density in Eq. (1) can be split into two terms as resolved and unresolved:

$$
\bar{\Sigma}=|\overline{\nabla c}|=\underbrace{\prod(\bar{c}, \bar{\Delta})}_{\text {Resolved }}+\underbrace{f(\bar{c}, \bar{\Delta}, \Pi(\bar{c}, \bar{\Delta}))}_{\text {Unresolved }}
$$

Where $(\bar{c})$ is the mean reaction progress variable, $(\bar{\Delta})$ is the filter width. The over-bar describes application of the spatial filter. The unresolved term in the above equation is evaluated using the following expression:

$$
\lambda=\bar{\Sigma}-\Pi(\bar{c}, \bar{\Delta})=|\overline{\nabla c}|-\Pi(\bar{c}, \bar{\Delta})
$$

Applying the test filter $\left({ }^{\wedge}\right)$ to flame surface density Eq. (2) leads to:

$$
\widehat{\bar{\Sigma}}=|\widehat{\nabla c}|=\underbrace{\prod(\hat{\bar{c}}, \hat{\bar{\Delta}})}_{\text {Resolved at test filter }}+\underbrace{[|\widehat{\nabla c}|-\Pi(\hat{\bar{c}}, \hat{\bar{\Delta}})]}_{\text {Unresolved at testfilter }}
$$

From the above equation, the unresolved flame surface density contribution at the test filter level can be written as:

$$
\Lambda=[|\widehat{\nabla c}|-\Pi(\widehat{\bar{c}}, \hat{\bar{\Delta}})]
$$

Assuming the sub-grid scale contribution of unresolved flame surface density at test filter is the same as that at grid filter and relating $(\lambda)$ and $(\Lambda)$ by using Germano identity [12]:

$$
\begin{gathered}
\Lambda-\hat{\lambda}=\left[|\widehat{\overline{\nabla c}}|-\prod(\hat{\bar{c}}, \hat{\bar{\Delta}})\right]-\left[|\widehat{\nabla c}|-\prod(\widehat{\bar{c}, \bar{\Delta}})\right] \\
\Lambda-\hat{\lambda}=[\Pi(\widehat{\bar{c}, \bar{\Delta}})-\Pi(\hat{\bar{c}}, \hat{\bar{\Delta}})]
\end{gathered}
$$

The sub-grid scale flame surface density contribution from the above equation can be added to the resolved flame surface density in Eq. (4) with a model coefficient (Cs) in order to obtain total flame surface density. Hence the flame surface density can be expressed as: 


$$
\bar{\Sigma}=\prod(\bar{c}, \bar{\Delta})+\operatorname{Cs}[\Pi(\widehat{\bar{c}, \bar{\Delta}})-\Pi(\hat{\bar{c}}, \hat{\bar{\Delta}})]
$$

The model coefficient $(C s)$ in above equation is dynamically obtained by identifying sub-grid scale flame surface as a fractal surface [4] as follows:

$$
C s=\frac{1}{1-\gamma^{2-D}}\left[\left(\frac{\bar{\Delta}}{\delta_{c}}\right)^{D-2}-1\right]
$$

Where $\left(\delta_{c}\right)$ is the lower cut-off scale, $(\lambda)$ is the ratio of test filter to grid filter, taken here equals to 2 and $(D)$ is the fractal dimension, calculated dynamically from [2].

$$
D=2.0+\frac{\log (\langle\Pi(\widehat{\bar{c}, \bar{\Delta}})\rangle /\langle\Pi(\hat{\bar{c}, \hat{\Delta}})\rangle)}{\log (\hat{\Delta} / \bar{\Delta})}
$$

The angular brackets ' $\langle$ ' in the previous equation indicates conditional averaging within the flame bounded by $\tilde{c}=0$ to $\tilde{c}=1$.

The other parameters used in the LES are summarized in the following table:

Table 1. Model parameters used in the present work

\begin{tabular}{|c|c|}
\hline Equivalence ratio & $\Phi=0.7$ \\
\hline Lower Heating Value $(\mathrm{kJ} / \mathrm{kmol})$ & 286,000 \\
\hline Laminar burning velocity $(\mathrm{m} / \mathrm{s})$ & 1.3 \\
\hline
\end{tabular}

The numerical model described above, has been implemented using a modified inhouse LES code [10]. The numerical results presented in this work are carried out using a grid independent resolution of $90 \times 90 \times 336$ (2.7 million) cells in 3 dimensional space (Figure 1c). The simulations are performed using an initially stagnant hydrogen-air mixture of equivalence ratio $\Phi=0.7$. Other mathematical details of the code are not described here but available in $[7,8]$.

\section{FLAME STRUCTURE}

Figure 2 shows a sequence of images for the development of the propagating flame at different times after ignition from the numerically obtained reaction rate contours compared with the measured LIF-OH images [9]. It is clear (not shown in experimental images), that the leading edge of the flame starts to expand hemispherically in the axial direction and the flame edge elongates in the radial direction. Once the flame hits the baffle plate, the laminar hemi-spherical structure is distorted and flame starts to expand through narrow vents. Accordingly, the surface area of the flame brush increases, hence, consumes more fuel/air mixture per unit time and propagates at relatively higher velocity through the un-burnt fuel/air mixture.

It is clear that the LES technique is capable of reproducing turbulent flame structure and the propagation rate with high level of accuracy. For instance at $4.2 \mathrm{~ms}$, both 
LES and measurements demonstrate the entrainment of un-burnt mixture on the upstream edge of the obstacle as it is surrounded by the flame. This trapped mixture is seen to burn between 4.2 and $4.4 \mathrm{~ms}$. However, there is some un-burnt mixture trapped in the formed recirculation zone downstream the solid obstacle and this burns after the leading edge of the flame has exited the chamber. It should be noticed here that experimental images are captured using the defined image tiers as shown in (Figure 1b) [9].
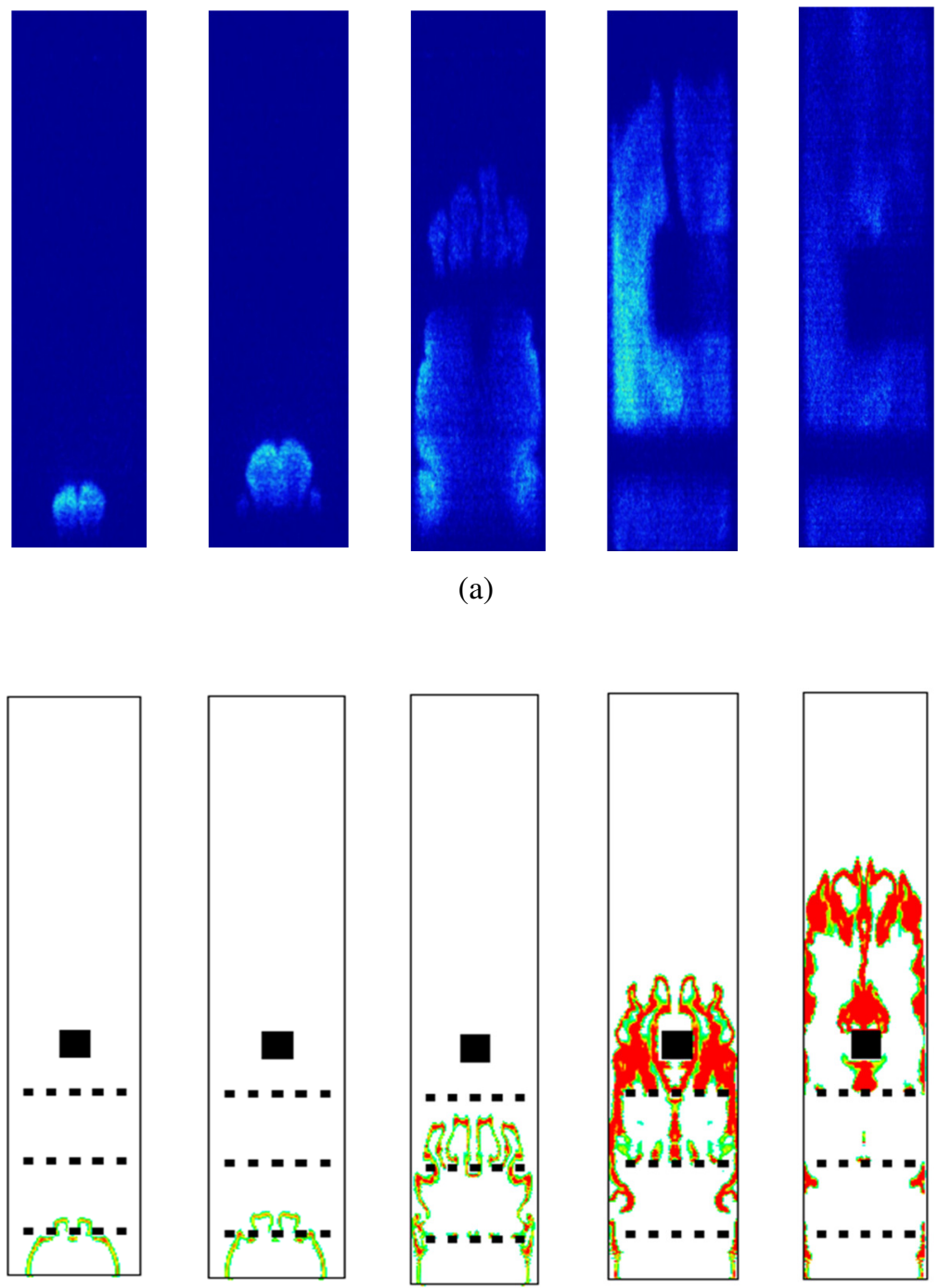

Reaction

Rate

$(\mathrm{Kg} / \mathrm{s})$

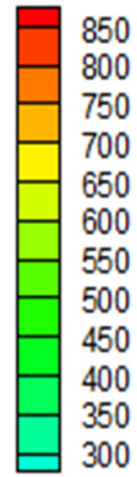

(b)
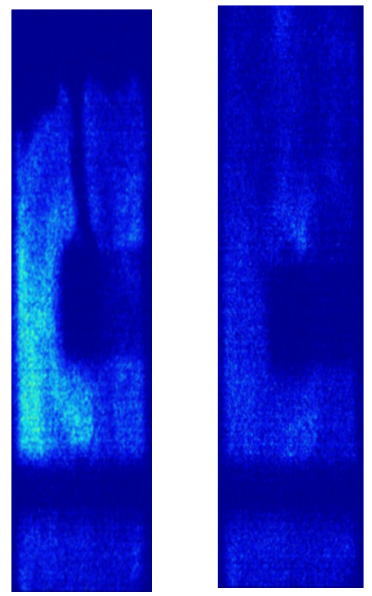

(a)

Fig. 2. Comparison between sequence of images showing flame structure after ignition.

(a) LIF-OH images from experiments [9]. (b) Numerical snapshots for reaction rate contours generated at 2.2, 2.4, 3.6, 4.2 and $4.4 \mathrm{~ms}$.

\section{RESULTS AND DISCUSSIONS}

Before going through the results in Figures 4 \& 5, an optimization for the ignition source is required. Hence, the analysis shown in Figure 3 is used to provide the most suitable ignition radius. It is found that the peak overpressure is independent of the ignition radius. The only effect found is in the timing of occurrence for the 
maximum pressure. The ignition radius which found to be most suitable to represent the early phase of flame propagation is $3 \mathrm{~mm}$. Also, for the same ignition area, the shape (i.e. hemi-spherical or spherical) has no effect on either the overpressure or the timing of occurrence, as both shapes have exactly overlapped (as for $3 \mathrm{~mm}$ case).

Figures 4 \& 5 compare between numerical and published experimental [9] results in terms of time histories for overpressure and flame position. It can be seen that the predicted overpressure shows encouraging comparisons against experimental data. The time traces in Figure 4 shows that, from LES the peak pressure prediction is about $721 \mathrm{mbar}$ and the experimental measurements is 778 mbar. The peak over pressure in LES and experiments correspond to the reconnection of the flame after the square obstacle and burning of trapped un-burnt gases down and upstream of the obstruction. It should be noted here that, the time shift between the experimental and LES is about $0.43 \mathrm{~ms}$. This discrepancy may be due to the difficulty in establishing the exact time of ignition in the experiments. However, in the LES predictions, ignition is initialized by setting reaction progress variable to 0.5 within a certain radius ( $3 \mathrm{~mm}$ radius is used here) at the start of the computations, i.e. time 0 ms. Figure 5 shows the comparison of predicted and measured time history of the flame position relevant to the ignition closed end. It can be seen that the agreement is remarkably good after some initial discrepancy. This again may be due to the uncertainty and modelling assumptions during the initial phase of flame propagation. These are to be further analysed and improved in further studies.

\section{CONCLUSION}

Overall, good agreements are obtained between the LES predictions and measurements for the case considered here. These kind of results encourage for further study and develop the work in hydrogen combustion and hence the applicability in more engineering applications. More extensive work is also required to validate the model for different flow and combustion configurations.

\section{REFERENCES}

[1] Charlette, F., C. Meneveau, and D. Veynante, "A power-law flame wrinkling model for LES of premixed turbulent combustion Part II: dynamic formulation", Combust. Flame, Vol. 131, pp. 181-197, (2002).

[2] Knikker, R., D. Veynante, and C. Meneveau, "A dynamic flame surface density model for large eddy simulation of turbulent premixed combustion", Phys. Fluids, Vol. 16, pp. L91-L94, (2004).

[3] Fureby, C., H. Pitsch, Lipatnikov, and E. Hawkes, "A fractal flame-wrinkling large eddy simulation model for premixed turbulent combustion", Proc. Combust. Inst., Vol. 30, pp. 593-601, (2005).

[4] Masri, A.R., S.S. Ibrahim, and B.J. Cadwallader, "Measurements and large eddy simulation of propagating premixed flames", Exp. Therm. Fluid Sci., Vol. 30, pp. 687-702, (2006).

[5] Pitsch, H., "Large-eddy simulation of turbulent combustion" Annu. Rev. Fluid Mech., Vol. 38, pp. 453-482, (2006). 
[6] Ranga Dinesh, K.K.J., X. Jiang, W. Malalasekera, and A. Odedra, "Large eddy simulation of fuel variability and flame dynamics of hydrogen-enriched nonpremixed flames", Fuel Process. Technol., Vol. 107, pp. 2-13, (2013).

[7] Gubba, S.R., S.S. Ibrahim, W. Malalasekera, and A.R. Masri, "LES modelling of propagating turbulent premixed flames using a dynamic flame surface density model", $2^{\text {nd }}$ ECCOMAS Thematic Conference on Computational Combustion, Delft, Netherlands, (2007).

[8] Ibrahim, S.S., S.R. Gubba, A.R. Masri, and W. Malalasekera, "Calculations of explosion deflagrating flames using a dynamic flame surface density model", J. Loss Prevent. Proc., Vol. 22, pp. 258-264, (2009).

[9] Masri, A.R., A. AlHarbi, S. Meares, and S.S. Ibrahim, "A Comparative Study of Turbulent Premixed Flames Propagating Past Repeated Obstacles", Ind. Eng. Chem. Res., Vol. 51, pp. 7690-7703, (2011).

[10] Kirkpatrick, M.P., S.W. Armfield, A.R. Masri, and S.S. Ibrahim, "Large Eddy Simulation of a Propagating Turbulent Premixed Flame", Flow Turbul. Combust. Vol. 70, pp. 1-19, (2003).

[11] lijima, T. and Takeno, T., "Effects of temperature and pressure on burning velocity", Combust. Flame, Vol. 65, No. 1, pp. 35-43, (1986).

[12] Germano, M., U. Piomelli, P. Moin, and W.H. Cabot, "A dynamic subgrid-scale eddy viscosity model”, Phys. Fluids A, Vol. 3, No. 7, pp. 1760-1765, (1991). 


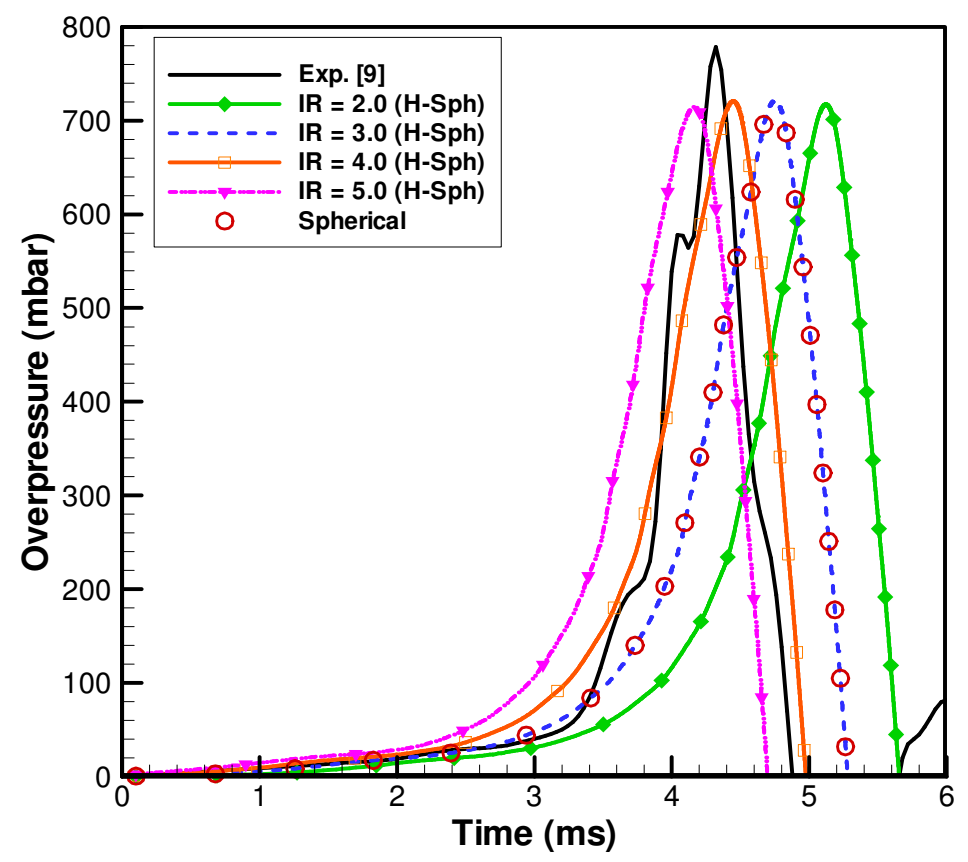

Fig. 3. Overpressure time traces of LES simulations using different ignition radii and shapes (IR: Ignition Radius, H-Sph: Hemi-Spherical shape) compared with experimental data [9].

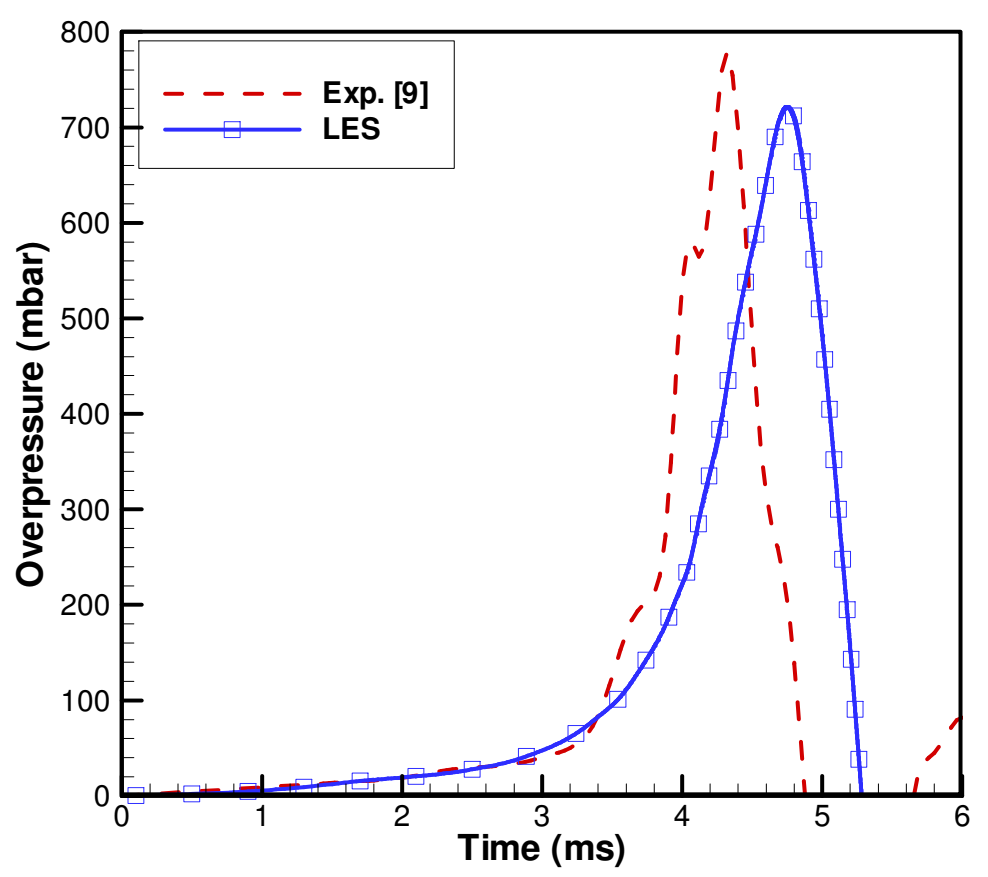

Fig. 4. Overpressure time traces of LES simulation compared with experimental data [9]. 


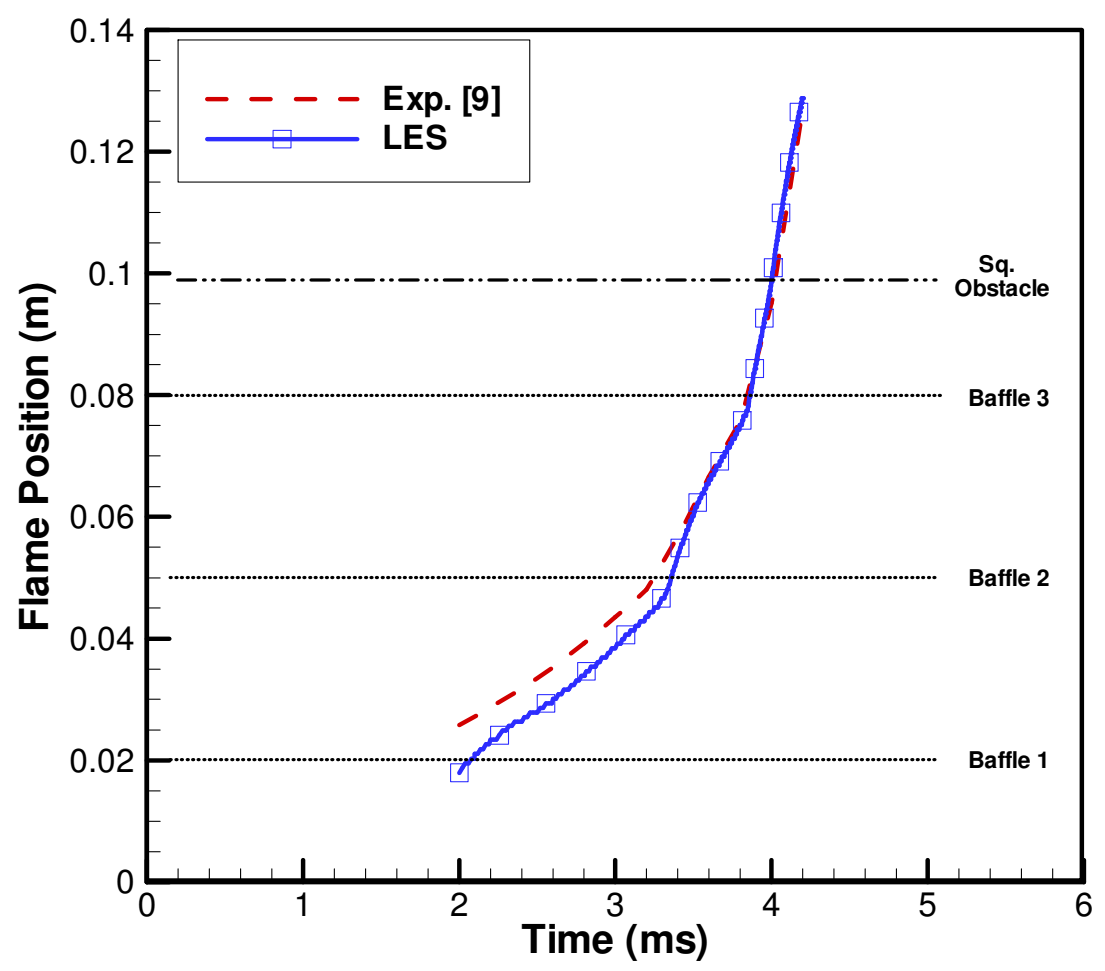

Fig. 5. Flame position time traces of LES simulation compared with experimental data [9]. 